\title{
Effect of CPAP therapy on daytime function in patients with mild sleep apnoea/hypopnoea syndrome
}

\author{
Heather M Engleman, Sascha E Martin, Ian J Deary, Neil J Douglas
}

\begin{abstract}
Background - Continuous positive airway pressure (CPAP) is an effective treatment in patients with moderate and severe sleep apnoea/hypopnoea syndrome (SAHS), but the minimum illness severity at which patients obtain benefit from CPAP is unclear. A study was therefore undertaken to investigate whether CPAP improves symptoms and daytime function in patients with mild SAHS.

Methods - Sixteen consecutively recruited patients with mild SAHS (5.0-14.9 apnoeas + hypopnoeas per hour slept and two or more symptoms of SAHS) participated in a prospective placebo controlled randomised crossover trial to assess the effects of CPAP on symptoms and daytime function. Patients spent four weeks on placebo and four weeks on CPAP, undergoing assessments of sleepiness, symptoms, cognitive performance, and well being on the last day of each treatment. Data from the placebo and CPAP assessments were compared.
\end{abstract}

Results - The mean (SE) objective effective use of CPAP was $2.8(0.7)$ hours per night. Significant improvements in symptom score $(-1.7(0.5), p<0.01)$, mental flexibility ( -14 (5) seconds, $p=0.02)$, and depression rating $(-1.6(0.8), p=0.03)$ on CPAP were observed. However, no significant differences in subjective or objective sleepiness were found. Ten of the 16 patients preferred CPAP and opted to continue with this treatment, although this proportion was non-significant $(p>0.4)$. The eight patients with best CPAP use showed an additional CPAP related improvement in quality of life $(-4.4(1.8)$, $\mathbf{p}=0.03)$. Those who complied better with CPAP therapy also had a higher average microarousal frequency $(p<0.01)$ and apnoea + hypopnoea index $(p=0.02)$ than the poorer compliers.

Conclusions - The results of this study provide evidence for improvements in symptoms and daytime function for patients with mild SAHS treated with CPAP.

(Thorax 1997;52:114-119)

Keywords: sleep apnoea/hypopnoea syndrome, CPAP, psychomotor performance.
Sleepiness and impaired daytime function are major clinical features of the sleep apnoea/ hypopnoea syndrome (SAHS) ${ }^{12}$ and the primary indication for treatment with continuous positive airway pressure (CPAP) is to try to improve these features. There is objective evidence that CPAP has beneficial effects on daytime sleepiness, cognitive performance, and psychological well being ${ }^{3-5}$ but these data have been gathered in patients with moderate or severe SAHS.

There is a dearth of data indicating whether patients with mild SAHS (5-15 apnoeas + hypopnoeas per hour slept with two or more symptoms of $\mathrm{SAHS}^{6}$ ) have abnormal objective daytime sleepiness or cognitive performance. A recent report, however, has suggested that patients with an apnoea + hypopnoea index (AHI) of less than 20 may have impaired quality of life. ${ }^{7}$ Many patients with mild SAHS seek treatment for their condition: $10 \%$ of CPAP users in a recent survey of patients attending a CPAP clinic had an AHI of $<15$ per hour slept. ${ }^{8}$ To establish whether such patients benefit from CPAP treatment we have carried out a prospective randomised placebo controlled study to examine the effect of treatment with CPAP on daytime function in patients with mild SAHS.

\section{Methods}

STUDY PROTOCOL

The study was constructed as a placebo controlled, balanced treatment order, crossover trial of daytime function in which 16 patients with mild SAHS spent four weeks on CPAP therapy (Sullivan APD-1 units, ResCare, Abingdon, UK) and four weeks on an oral placebo (Ranitidine $300 \mathrm{mg}$ homologue, Glaxo, Greenford, UK) in a dose of two tablets at bedtime. Patients were educated in the mechanisms of action of CPAP therapy and were asked to use their CPAP units all night, most especially on the night before assessment. With the permission of the local ethics subcommittee, patients were told that the placebo tablets might improve upper airway function. There was no washout period between treatments. Assessments of daytime function were performed on the last day of each treatment. Eight patients commenced treatment with CPAP and eight with placebo. 
Table 1 Daytime function assessments

\begin{tabular}{ll}
\hline Sleepiness & Multiple sleep latency test (MSLT) \\
& UWIST mood adjective checklist (UMACL) \\
Epworth sleepiness scale & Single score for eight-item questionnaire \\
Symptoms & (snoring, choking in sleep, morning headaches, \\
& morning confusion, wakening during the night, \\
& daytime napping, evening napping, sleepiness whilst \\
& driving) \\
& WAIS-R performance IQ \\
& (Block Design and Digit Symbol Substitution) \\
& National Adult Reading Test (NART) \\
TrailMaking & 30 minute SteerClear \\
& Paced Auditory Serial Addition \\
& (PASAT: two second presentation rate) \\
& Rapid Visual Information Processing (RVIP) \\
& Eight-choice reaction time \\
& Verbal Fluency \\
& Benton Visual Retention Test (BVRT) \\
& Hospital Anxiety and Depression Scale (HADS) \\
& General Health Questionnaire-28 (GHQ-28) \\
& Nottingham Health Profile (NHP) Part 2 \\
& \\
&
\end{tabular}

WAIS-R $=$ Wechster adult intelligence scale (revised)

Before the commencement of treatment patients attended a session of familiarisation with the psychometric tests used in assessment to minimise learning effects across subsequent assessments. Before treatment subjects also underwent a CPAP titration study to establish the therapeutic pressure at which hypopnoeas and electroencephalographic arousals from sleep were minimised. Patients were contacted two weeks into the CPAP treatment limb to check progress and were encouraged to report any problems with CPAP use so that these could be managed and compliance optimised. Subjects were asked to avoid caffeinated beverages on the assessment days and were offered only decaffeinated refreshments.

PATIENTS

Subjects were prospectively recruited from consecutive outpatients referred to the Sleep Clinic for investigation of SAHS. Entry criteria required two or more symptoms of SAHS ${ }^{1}$ and an $\mathrm{AHI}$ in the range 5.0-14.9 per hour slept during clinical polysomnography, conducted and scored according to our usual methods. ${ }^{26}$ Polysomnography included electroencephalographic (EEG), electro-oculographic (EOG), and electromyographic (EMG) monitoring to facilitate the evaluation of sleep quality and microarousals (defined by increases in EEG frequency of 1.5 seconds or longer, coincident with any duration of increased EMG activity ${ }^{2}$ ). Breathing pauses were monitored by inductance plethysmography of abdominal and thoracic respiratory movement and by thermistor assessment of oronasal airflow. Hypopnoeas were defined as 10 seconds or longer of $50 \%$ reduction in respiratory movement and apnoeas as 10 seconds or longer of absent airflow. ${ }^{6}$ Arterial oxygen saturation was monitored using pulse oximetry. Patients with coexisting neurological or sleep disorders, or residence outwith a 50 mile radius of the laboratory, were excluded.

Twenty eight subjects were invited to participate in the study, 18 of whom accepted. Of the 10 non-acceptors, four declined to par- ticipate because work pressure meant they could not spend the required three full days in the sleep laboratory, four gave no reason, and two were intolerant of nasal CPAP during the pretreatment CPAP titration trial. Two patients withdrew after the start of the study, both during the CPAP limb, and were excluded from the analysis. One of these moved away unexpectedly and remains on CPAP therapy, while the other was intolerant of noise from his CPAP unit and declined to complete the treatment limb. The treatment order slots of patients withdrawing after the start of the study were filled with the next available subject.

The 16 patients $(12 \mathrm{men})$ who took part in the study had a mean (SE) age of 52 (2) years with a mean body mass index of 29.8 (1.8) $\mathrm{kg} / \mathrm{m}^{2}$. The subjects had a mean AHI of 11 (1) per hour slept, the mean number of microarousals per hour slept was 24 (3), mean minimum oxygen saturation was $86(1) \%$, and the mean $4 \%$ desaturation rate was 4 (1) per hour slept. The mean baseline Epworth sleepiness score was 14 (1) in the nine patients in whom it was available, and all 16 patients complained of both snoring and regular napping behaviour as symptoms.

\section{ASSESSMENTS}

Daytime function assessments

Daytime function assessments as previously described and referenced ${ }^{5}$ were obtained on the last day of each treatment. Tests (table 1) were administered in a standardised order to control for circadian effects. Symptoms were rated as present or absent using an eight point questionnaire and sleepiness was assessed using the multiple sleep latency test $\left(\right.$ MSLT) ${ }^{9}$ and the energetic arousal scale of the UWIST mood adjective checklist (UMACL). The Epworth Sleepiness Scale, ${ }^{1011}$ a self-rating of recent sleepiness behaviour, was obtained in addition to the other assessments in nine patients. The cognitive tests covered a wide range of function known to be affected by SAHS, including vigilance, attention and concentration, visuomotor skill, verbal fluency, and memory. The premorbid level of cognitive function was estimated at the familiarisation session using the National Adult Reading Test (NART), while the current level of general intellectual function was measured with the Digit Symbol Substitution and Block Design subtests from the WAIS-R battery and with TrailMaking task B. The NART score provided an estimate of the premorbid level of function while the WAIS$\mathrm{R}$ subtests allowed a measurement of current level of function, the performance IQ (PIQ), to be made. By subtracting the assessed WAISR PIQ on each treatment from the NART estimate of premorbid PIQ an "IQ decrement score" was obtained. Tests of attention included a 30 minute driving-based vigilance task (SteerClear), Paced Auditory Serial Addition Test (PASAT) at two second presentation rate, the Rapid Visual Information Processing Test (RVIPT), and eight choice reaction time. Verbal fluency was assessed with Borkowski's test and memory with the Benton Revised 

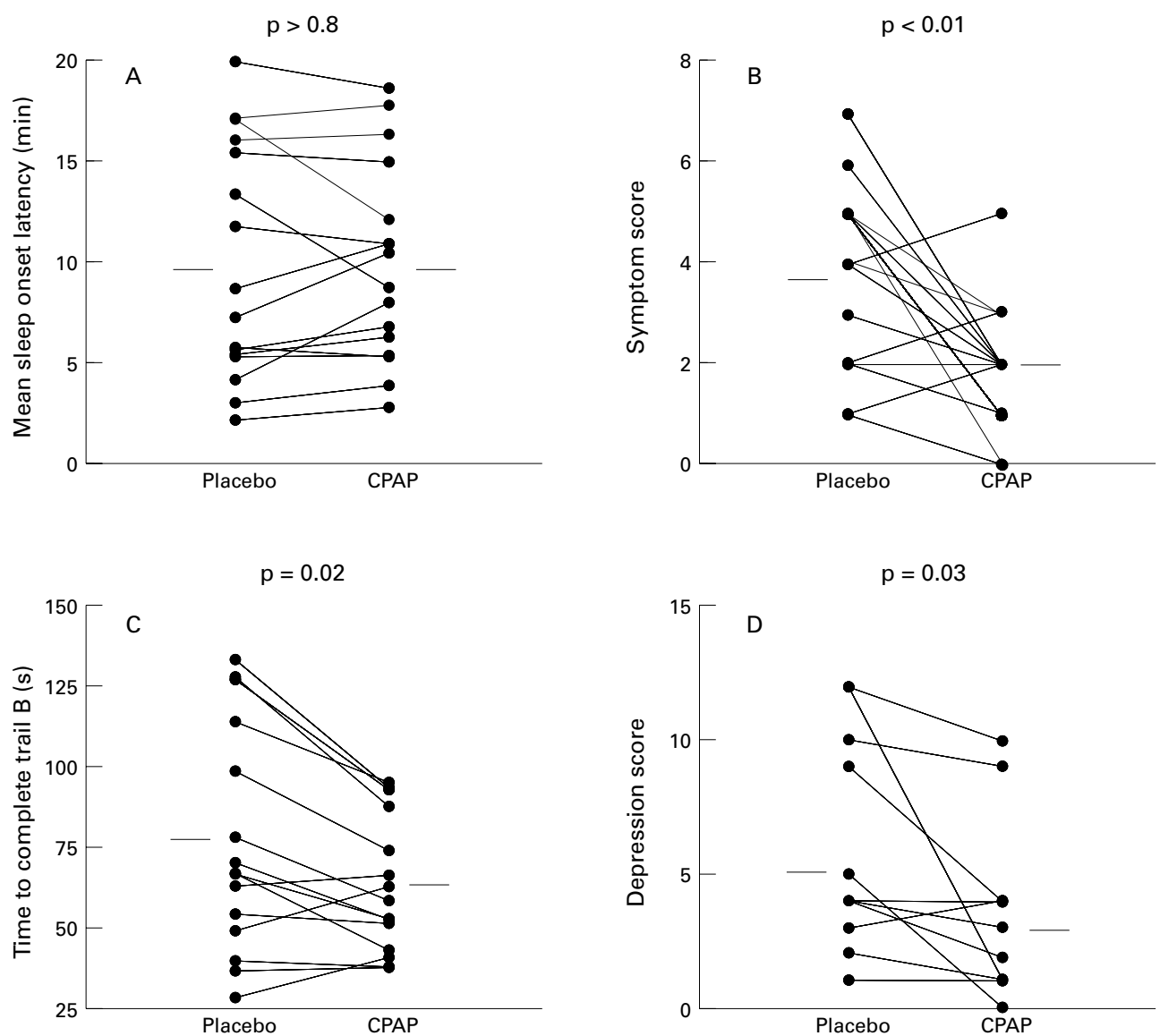

Figure 1 Values on placebo and treatment with CPAP for (A) mean sleep onset latency, (B) symptom score, $(C)$ TrailMaking $B$ performance, and (D) HADS depression rating.

Visual Retention Test (BVRT). Alternative forms of the letters employed in the verbal fluency test and of the designs used in the BVRT were administered in succeeding assessment sessions. Minor psychiatric morbidity was self-rated on the Hospital Anxiety and Depression Scale (HADS) and the General Health Questionnaire-28 (GHQ-28), and quality of life on Part 2 of the Nottingham Health Profile (NHP). At the final assessment subjects were asked to rate which treatment they preferred, weighing ease of use and treatment benefit.

CPAP compliance

Compliance with CPAP therapy was monitored by reading time clocks ${ }^{12}$ hidden within the casing of the CPAP units which measured the total duration during the treatment limb that units were switched on and that CPAP was delivered effectively to the nasal mask. Mean compliance rates for the CPAP treatment period (hours per night) were calculated.

ANALYSIS OF DATA

Outcome measurements of daytime function from placebo and CPAP assessments were compared. Continuous normally distributed data were examined using two-way ANOVA with treatment order as a between subjects factor, treatment type as a within subject factor, and the interaction between these two factors representing learning effects. This analysis, in conjunction with the study design, allows learning effects to be factored out from treatment effects. Paired Wilcoxon tests were conducted for interval and non-normal data. The binomial test was used to examine the distribution of treatment preference, and $\mathrm{McNemar}$ tests to assess changes in frequency of individual symptoms. Patients were split into better and poorer compliers using median effective CPAP use as a cutoff point. A subanalysis comparing CPAP and placebo outcome measurements was conducted within the group that complied better with CPAP treatment. Mann-Whitney tests were conducted to compare background variables in the better and poorer compliers with CPAP treatment. All statistical analyses employed two tailed $\mathrm{p}$ values and were performed using SPSS-PC.$+{ }^{13}$

\section{Results}

FULL GROUP

CPAP units were run for a mean (SE) of 3.2 (0.7) hours per night and used effectively for 2.8 (0.6) hours per night.

The measurements obtained while receiving treatment with CPAP were compared with those obtained on placebo treatment. Neither objective daytime sleepiness measured by the 
Table 2 Mean (SE) effects of placebo and CPAP treatment on daytime function

\begin{tabular}{|c|c|c|c|c|c|c|}
\hline & \multicolumn{2}{|l|}{ Full group } & \multirow[b]{2}{*}{$p$ value } & \multicolumn{2}{|c|}{ Better CPAP compliers } & \multirow[b]{2}{*}{$p$ value } \\
\hline & Placebo & $C P A P$ & & Placebo & $C P A P$ & \\
\hline Mean sleep onset latency (min) & $9.9(1.5)$ & $10.0(1.2)$ & NS & $10.8(2.3)$ & $11.0(2.1)$ & NS \\
\hline UMACL energetic arousal score & $22.7(1.7)$ & $25.4(1.4)$ & NS & $23.0(2.8)$ & $27.8(1.6)$ & NS \\
\hline Epworth sleepiness score & $10.0(1.2)$ & $10.1(1.4)$ & NS & $6.7(1.5)$ & $7.3(3.5)$ & NS \\
\hline Symptom score $†$ & $3.7(0.4)$ & $2.0(0.3)$ & $<0.01$ & $3.6(0.8)$ & $1.5(0.3)$ & 0.02 \\
\hline IQ decrement score & $5.3(3.5)$ & $7.0(3.1)$ & NS & $5.9(5.4)$ & $7.0(4.4)$ & NS \\
\hline TrailMaking B (secs)† & $77.7(9.2)$ & $64.1(5.5)$ & 0.02 & $76.1(14.1)$ & $61.9(9.1)$ & 0.04 \\
\hline SteerClear (obstacles hit) & $75.3(8.9)$ & $74.8(7.3)$ & NS & $83.7(13.1)$ & $81.7(12.1)$ & NS \\
\hline PASAT (2 sec rate) (correct) & $35.3(2.8)$ & $37.8(3.3)$ & NS & $33.3(4.1)$ & $37.4(5.7)$ & NS \\
\hline RVIPT (correct) & $34.8(3.2)$ & $36.9(3.2)$ & NS & $36.6(4.0)$ & $34.4(3.5)$ & NS \\
\hline Median eight-choice reaction time (ms) & $356(14)$ & $365(16)$ & NS & $365(18)$ & $386(25)$ & NS \\
\hline Verbal fluency (total words) & $39.2(3.1)$ & $38.5(3.5)$ & NS & $36.7(5.4)$ & $35.0(5.8)$ & NS \\
\hline BVRT (correct) $\dagger$ & $7.3(0.6)$ & $7.3(0.6)$ & NS & $7.1(0.7)$ & $7.1(0.9)$ & NS \\
\hline HADS anxiety score $†$ & $5.1(1.1)$ & $4.5(1.2)$ & NS & $4.5(1.7)$ & $3.9(1.8)$ & NS \\
\hline HADS depression score $†$ & $5.0(1.0)$ & $3.4(0.9)$ & 0.03 & $4.8(1.5)$ & $2.3(1.0)$ & 0.04 \\
\hline GHQ-28 total score $\dagger$ & $3.2(1.3)$ & $1.4(0.6)$ & NS & $3.3(0.1)$ & $0.4(0.3)$ & NS \\
\hline NHP Pt 2 total score $\dagger$ & $5.8(1.4)$ & $3.8(1.1)$ & NS & $6.8(2.5)$ & $2.4(1.5)$ & 0.03 \\
\hline
\end{tabular}

UMACL = UWIST mood adjective checklist; PASAT = Paced auditory serial addition test; RVIPT = Rapid visual information processing test; BVRT $=$ Benton visual retention test; HADS $=$ Hospital anxiety and depression scale; GHQ-28 $=$ General health questionnaire-28; NHP $=$ Nottingham health profile; $\mathrm{NS}=$ non-significant $(\mathrm{p}>0.05)$.

t Mann-Whitney test.

MSLT ( $p>0.8$ (ANOVA); fig 1A) nor subjective sleepiness measured on the Epworth scale were significantly improved by CPAP ( $>>0.9$ (ANOVA); table 2). The total symptom score (fig 1B) was significantly lower on CPAP than placebo ( $p<0.01$, Wilcoxon), reflecting an improvement in status. Exclusion of the snoring symptom item from the total resulted in a nonsignificant improvement in symptom total from treatment with placebo $(2.8(0.5))$ to treatment with CPAP (1.9 (0.3); $\mathrm{p}=0.16)$. Performance on the TrailMaking B task of mental flexibility was significantly improved on CPAP $(p=0.02$ (Wilcoxon); fig 1C) but no other treatment effects on cognitive performance were found. Self-rated depression score on the Hospital Anxiety and Depression Scale (HADS) was significantly reduced by CPAP ( $p=0.03$ (Wilcoxon); fig 1D), corresponding to lower distress. Ten of the 16 patients preferred CPAP when the factors of ease of use and treatment benefit were weighed $(p>0.4)$, and these patients commenced on long term treatment with CPAP at the conclusion of the study.

The effects of treatment order on the variables examined were non-significant $(p>0.12)$ with the exception of mean sleep onset latency $(p=0.05)$ which did not show a significant treatment effect. Significant treatment $\times$ treat- ment order interactions, indicating learning effects, were demonstrated for SteerClear performance $(p<0.001)$, the WAIS-R performance IQ score $(\mathrm{p}=0.05)$, and verbal fluency score $(\mathrm{p}<0.01)$

BETTER COMPLIERS WITH CPAP

The patient group was split by median effective CPAP use into better and poorer compliers ( $n=8$ in each group). The mean (SE) effective CPAP use was $5.0(0.6)$ hours per night in the more compliant users and $1.1(0.2)$ hours per night in the poorer users. Four patients commenced treatment with tablets in each group. As in the full group, those who complied better with CPAP treatment showed significant CPAP-related improvement in symptom total score $(\mathrm{p}=0.02)$, TrailMaking $B$ performance $(\mathrm{p}=0.04)$, and HADS depression rating $(\mathrm{p}=$ 0.04 ; table 2 ). In addition, the NHP part 2 score was significantly reduced in patients on CPAP $(\mathrm{p}=0.03)$, indicating improved quality of life. Six of the eight better compliers preferred CPAP treatment $(p>0.2)$.

The background features of the better and poorer compliers with CPAP were compared to assess predictors of greater CPAP use. The variables that were compared included poly-
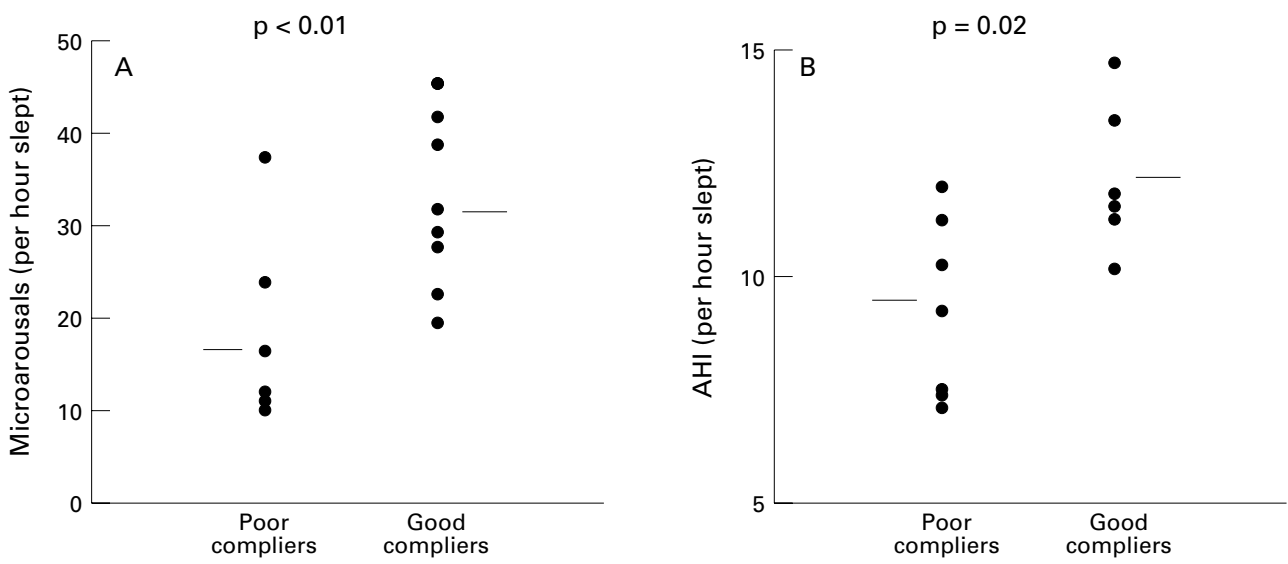

Figure 2 (A) Microarousal index and (B) apnoea + hypopnoea index (AHI) in poor and better compliers with CPAP treatment. 
Table 3 Mean (SE) differences in background features of poor and better compliers with CPAP

\begin{tabular}{lccc}
\hline & Poor compliers & Better compliers & p value \\
\hline AHI (per hour slept) & $9.6(0.7)$ & $12.5(0.5)$ & 0.02 \\
Microarousal index (per hour slept) & $16.6(3.4)$ & $32.2(3.2)$ & $<0.01$ \\
Minimum $\mathrm{O}_{2}$ saturation (\%) & $87(1)$ & $85(2)$ & $\mathrm{NS}$ \\
4\% desaturations (per hour slept) & $2.3(1.1)$ & $5.3(1.7)$ & $\mathrm{NS}$ \\
Symptom score & $5.1(0.2)$ & $4.8(0.8)$ & $\mathrm{NS}$ \\
Epworth sleepiness score & $13.8(1.1)$ & $15.0(2.0)$ & $\mathrm{NS}$ \\
UMACL Energetic arousal score & $22.6(2.4)$ & $21.1(2.2)$ & $\mathrm{NS}$ \\
Mean sleep onset latency on placebo (min) & $9.0(2.0)$ & $10.8(2.3)$ & $\mathrm{NS}$ \\
\hline
\end{tabular}

$\mathrm{AHI}=$ apnoea + hypopnoea index; $\mathrm{UMACL}=\mathrm{UWIST}$ mood adjective checklist; $\mathrm{NS}=$ not significant $(\mathrm{p}>0.05)$. somnographic measurements, symptoms and sleepiness reported at familiarisation, and sleep onset latency when off CPAP (table 3). Both microarousal index $(\mathrm{p}<0.01)$ and AHI $(\mathrm{p}=$ 0.02 ) were significantly higher in those who complied better with CPAP. However, there was considerable overlap between better and poorer compliers in ranges for both microarousal index (fig 2A) and AHI (fig 2B). There was no difference in the pretreatment symptom score between poor and good compliers, and no difference in sleepiness measurements when off CPAP between the two groups.

\section{Discussion}

The results of this study suggest that patients with mild SAHS gain benefit for symptoms, cognitive performance, and psychological distress from CPAP. We have previously reported that CPAP improves these same measurements of daytime function in patients with more severe SAHS. ${ }^{5}$ Thus, the study extends previous findings on the therapeutic value of CPAP to apply to symptomatic patients with 5-15 apnoeas + hypopnoeas per hour of sleep, and indicates that such patients should be given a trial of CPAP therapy.

Learning effects were demonstrated on some cognitive performance tests, highlighting the importance of controlling for learning in longitudinal, treatment related studies. However, the lack of effect of treatment order on variables improved by treatment with CPAP ensured that the balancing of treatment order employed within this crossover study could control for such learning effects.

Those daytime function tests that showed improvement in patients with mild SAHS symptoms, cognitive performance and depression ratings - were also improved by CPAP in a placebo controlled study of daytime function in patients with SAHS of a wider range of severity, ${ }^{5}$ confirming the sensitivity of these measurements to the effects of CPAP. In patients with mild SAHS and higher levels of CPAP compliance, improvement in quality of life was observed with CPAP treatment in our patients as well as in those with SAHS of a wider range of severity. ${ }^{5}$ Most of the patients preferred the CPAP treatment and opted to continue with it after the end of the study.

However, by no means all the tests of function administered showed improvements in patients on CPAP. This may reflect marginal or selective cognitive impairment in mild
SAHS, or may also relate to the difficulties in detecting sleepiness induced deficits with cognitive tests. ${ }^{14}$ It is notable that the Epworth score was no more responsive to CPAP than to placebo treatment, although the reduced sample size of nine patients limited statistical power to show change. Both placebo and CPAP significantly reduced Epworth scores from a baseline level of $14(1)(p<0.03)$, confirming the strength of placebo effects.

A potential problem with this study is the multiple outcome measurements analysed, raising the possibility of a type 1 false positive statistical error. Such errors occur at a frequency of one in 20 comparisons. In our study 16 tests were employed and thus three significant comparisons would not be expected by chance alone. Furthermore, all three significant findings were in the direction of improvement with CPAP - the hypothesised direction. We have used two tailed rather than one tailed $p$ values to adopt a conservative statistical approach. In addition, the improvements with CPAP are consistent with our previous findings in patients with a wider range of severity of SAHS. ${ }^{5}$ We accept that further data will be required to confirm these observations. The current study will allow refinement of techniques for such studies by identification of the relevant tests.

Neither objective (MSLT) nor subjective (Epworth scale) sleepiness was improved by CPAP in patients with mild SAHS. Mean sleep onset latency approached the normal range on both placebo and $\mathrm{CPAP}^{9}$ while subjective sleepiness scores were increased on both placebo and CPAP. ${ }^{11}$ The results with the MSLT contrast with those from studies of patients with "upper airway resistance syndrome" (UARS) ${ }^{1516}$ whose short sleep onset latencies, indicative of moderate or severe sleepiness before treatment, were extended into the normal range by CPAP. Recent studies with CPAP in patients with more severe SAHS have shown statistical improvement but a lack of normalisation in objective daytime sleepiness with CPAP. ${ }^{3-5}$ Thus, the usefulness of the MSLT in treatment related studies may be questioned. Alternative polysomnographic tests of daytime sleepiness, such as the maintenance of wakefulness test which more explicitly assesses the ability to stay awake, may, as has been suggested, ${ }^{17}$ prove more sensitive to treatment effects.

The cognitive task showing improvement with CPAP, TrailMaking B, is a test of mental flexibility requiring rapid and complex responses. Performance on this test is impaired in patients with severe SAHS $^{318}$ and was previously found to be unimproved following CPAP therapy in a sample of 10 patients with SAHS. ${ }^{3}$ The indication of CPAP related improvement in TrailMaking B performance in the current study may reflect improved statistical power with its larger sample. Alternatively, cognitive deficits may be more reversible in patients with milder SAHS in whom sleep fragmentation rather than hypoxaemia may play the more important role in determining cognitive impairment. 
Our study was carried out in patients with mild SAHS who had 5-15 apnoeas + hypopnoeas per hour slept, using our standard definitions for apnoeas ${ }^{19}$ and hypopnoeas. ${ }^{6}$ Our data cannot be extrapolated to patients with UARS, ${ }^{16}$ although it is possible that some centres will diagnose patients who satisfy our criteria for mild SAHS as having UARS. We suspect that this is particularly a problem with the definition of hypopnoea which varies between centres and with different sensors. ${ }^{620}$

An earlier study of CPAP administration in 15 snorers with AHI of $<5$ found that no subjects opted for long term treatment. ${ }^{21}$ In this study 10 of the 16 patients (and six of eight of the better compliers) preferred CPAP and opted for home CPAP therapy. The discrepancy between acceptance of CPAP treatment in these two studies is likely to relate to the symptomatology of the patient samples. All subjects in the current study complained of two or more symptoms of SAHS, one of which was regular napping behaviour, while the level of daytime problems in the previous study was low.

The low objectively monitored compliance rates with CPAP might be considered disappointing, but are similar to those observed in other prospective studies of early CPAP compliance rates in new users. ${ }^{51322}$ Although clinicians recommend all night CPAP use, there has been little research on the relationship between rate of use and benefit. As in the case of patients with the UARS ${ }^{23}$ or snorers with an AHI of $<10,{ }^{24}$ patients with mild SAHS are likely to show proportionately less disturbed sleep than those with more severe SAHS. Such patients may thus require shorter CPAP administration each night to acquire the "core sleep" required for normal daytime function. ${ }^{25}$

A potential criticism of this study is the short duration of the study which involved eight weeks of participation, with four weeks of CPAP therapy. A previous study has shown improvement in objective daytime sleepiness after as little as two nights on CPAP. ${ }^{26} \mathrm{We}$ identified improvement in function after four weeks on CPAP in patients with mild SAHS, as had previously been shown in more severe patients. ${ }^{5}$

Examination of the background differences between good and poor compliers with CPAP treatment revealed evidence of greater microarousal frequency and higher AHI in the better compliers, suggesting that microarousal frequency more strongly determines CPAP use than AHI, the more commonly employed measurement of severity of SAHS. This empirical finding supports the role of sleep fragmentation in inducing daytime deficits. ${ }^{21524}$ However, neither symptoms nor sleepiness were predictive of the level of CPAP use.

We conclude that treatment with CPAP improves symptoms and daytime function in patients with mild SAHS. A trial of CPAP therapy is therefore clinically indicated for symptomatic patients with 5-15 apnoeas + hypopnoeas per hour of sleep. However, further controlled studies in this patient group are required to help identify whether the improvements in daytime function are sustained, to identify whether other clinical features of SAHS are improved by CPAP, and to help predict which patients will use CPAP.

The authors thank Glaxo for their provision of placebo tablets and ResCare for their donation of CPAP units for use in this study. We gratefully acknowledge the contributions of the nursing, technical, and administrative staff of the Scottish National Sleep Laboratory to this project. Dr $\mathrm{H} \mathrm{M}$ Engleman is supported by a grant from the British Lung Foundation.

1 Whyte KF, Allen MB, Jeffrey AA, Gould GA, Douglas NJ Clinical features of the sleep apnoea/hypopnoea syndrome. $Q \mathcal{F}$ Med 1989;72:659-66.

2 Cheshire K, Engleman H, Deary I, Shapiro C, NJ Douglas Factors impairing daytime performance in patients with sleep apnea/hypopnea syndrome. Arch Intern Med 1992; 152:538-41.

3 Bédard M-A, Montplaisir J, Malo J, Richler F, Rouleau I. Persistent neuropsychological deficits and vigilance impairment in sleep apnea syndrome after treatment with continuous positive airways pressure (CPAP). 7 Clin Exp Neuropsychol 1993;15:330-41.

4 Kribbs NB, Pack AI, Kline LR, Getsy JE, Schuett JS, Henry JN, et al. Effects of one night without nasal CPAP treatment on sleep and sleepiness in patients with ob-

En effeman HM, Martin SE, Deary 1J, Douglas NJ. The effect of conthuous positive airway pressure therapy on daytime function in the sleep apnoea/hypopnoea syndrome. Lancet 1994:343:572-5.

6 Gould GA, Whyte KF, Rhind GB, Airlie MAA, Catterall JR, Shapiro CM, et al. The sleep hypopnea syndrome. Am Rev Respir Dis 1988;137:895-8.

7 Gall R, Isaac L, Kryger M. Quality-of-life in mild obstructive sleep apnea. Sleep 1993;16:S59-61

8 Engleman HM, Asgari-Jirhandeh N, McLeod AL, Ramsay CF, Deary IJ, Douglas NJ. Self-reported use of CPAP and benefits of CPAP therapy: a patient survey. Chest 1996 109:1470-6.

9 Thorpy MJ. The clinical use of the multiple sleep latency test. Sleep 1992;15:268-76.

10 Johns MW. A new method for measuring daytime sleepiness: the Epworth sleepiness scale. Sleep 1991;14:540-5.

11 Johns MW. Daytime sleepiness, snoring and obstructive sleep apnea: the Epworth sleepiness scale. Chest 1993; 103:30-6.

12 Reeves-Hoché MK, Meck R, Zwillich CW. Nasal CPAP. an objective evaluation of patient compliance. Am $\mathcal{F}$ Respir Crit Care Med 1994;149:149-54.

13 Norusis MJ/SPSS Inc. SPSS/PC + advanced users guide. Chicago: SPSS Inc, 1988

14 Wilkinson RT, Edwards RS, Haines E. Performance following a night of reduced sleep. Psychon Sci 1966;3:471-2.

5 Guilleminault C, Stoohs R, Clerk A, Simmons J, Labanowski $M$. From obstructive sleep apnea syndrome to upper airway resistance syndrome: consistency of daytime sleepiness. Sleep 1992;15:S13-16.

16 Guilleminault C, Stoohs R, Clerk A, Cetel M, Maistros P. A cause of excessive daytime sleepiness: the upper airway resistance syndrome. Chest 1993;104:781-7.

17 Sangal RB, Thomas L, Mitler MM. Maintenance of wakefulness test and multiple sleep latency test: measurement of different abilities in patients with sleep disorders. Chest 1992;101:898-902.

18 Findley LJ, Barth JT, Powers DC, Wilhoit SE, Boyd DG Surratt PM. Cognitive impairment in patients with obstructive sleep apnea and associated hypoxemia. Chest 1986;90:686-90.

19 Guilleminault C, van den Hoed J, Mitler MM. Clinical overview of the sleep apnea syndromes. In: Guilleminault C, Dement WC, eds. Sleep apnea syndromes. New York: Alan R Liss, 1978: 1-12.

20 Moser NJ, Phillips BA, Berry DTR, Harbison L. What is hypopnea, anyway? Chest 1994;105:426-8.

21 Guilleminault C, Stoohs R, Duncan S. Snoring (I): daytime sleepiness in regular heavy snorers. Chest 1991;99:44-8.

22 Kribbs NB, Pack AI, Kline LR, Smith PL, Schwartz AR, Schubert NM, et al. Objective measurement of pattern of nasal CPAP use by patients with obstructive sleep apnea. Am Rev Respir Dis 1993;147:887-95.

23 Downey R, Perkins RM, McQuarrie J. Upper airway resistance syndrome; sick, symptomatic and underrecognized. Sleep 1993;16:620-3.

24 Zucconi M, Oldani A, Ferini-Strambi L, Calori G, Castronovo C, Smirne S. EEG arousal pattern in habitual snorers with and without obstructive sleep apnoea (OSA). Snorers with and without obsti

25 Horne J. Why we sleep. New York: Oxford University Press, 1988

26 Lamphere J, Roehrs T, Wittig R, Zorick F, Conway WA Roth T. Recovery of alertness after CPAP in apnea. Chest 1989;96:1364-7. 